OCHA-PP-131

STUPP-99-156

hep-th/9904010

April, 1999

\title{
Super Weyl Anomalies in the AdS/CFT Correspondence
}

\author{
MAdOKA Nishimura \\ Department of Physics, Ochanomizu University \\ 2-1-1, Otsuka, Bunkyo-ku, Tokyo 112-0012, Japan \\ and \\ YOSHIAKI TANII \\ Physics Department, Faculty of Science \\ Saitama University, Urawa, Saitama 338-8570, Japan
}

\begin{abstract}
Anomalies of $N=(4,4)$ superconformal field theories coupled to a conformal supergravity background in two dimensions are computed by using the AdS/CFT correspondence. We find that Weyl, axial gauge and super Weyl transformations are anomalous, while general coordinate, local Lorentz, vector gauge and local super transformations are not. The coefficients of the anomalies show that the superconformal field theories have the central charge expected in the AdS/CFT correspondence.
\end{abstract}

* e-mail: tanii@post.saitama-u.ac.jp 


\section{Introduction}

According to the AdS/CFT correspondence [1, 2, 3] the string/M-theory in $(d+$ 1)-dimensional anti de Sitter (AdS) space times a compact space is dual to a $d$ dimensional conformal field theory (CFT) defined on the boundary of the AdS space. There is a one-to-one correspondence between boundary values $\phi_{0 I}(x)$ of bulk fields $\phi_{I}(x)$ and operators $\mathcal{O}_{I}(x)$ in the boundary CFT. The generating functional of correlation functions of operators $\mathcal{O}_{I}$ in the CFT is given by the partition function of the string/M-theory. In a certain limit the string/M-theory is approximated by a low energy effective supergravity and the partition function is given by a stationary point of the supergravity action $S[\phi]$

$$
\left\langle\exp \left(i \int d^{d} x \sum_{I} \phi_{0 I}(x) \mathcal{O}_{I}(x)\right)\right\rangle_{\mathrm{CFT}}=\exp (i S[\phi]) .
$$

Here, $\phi_{0 I}$ on the left hand side are arbitrary functions defined on the $d$-dimensional boundary while $\phi_{I}$ on the right hand side are the solutions of supergravity field equations in the bulk satisfying boundary conditions $\phi_{I}=\phi_{0 I}$.

The boundary fields $\phi_{0 I}$ are expected to form supermultiplets of a $d$-dimensional conformal supergravity [4, 5] since they couple to CFT operators. In ref. [6] we studied this relation between AdS supergravities in $d+1$ dimensions and conformal supergravities in $d$ dimensions in the case of three-dimensional $(p, q)$ AdS supergravities based on the superalgebra $\operatorname{OSp}(p \mid 2) \times \operatorname{OSp}(q \mid 2)$ [7]. We explicitly showed that the local symmetry transformations of the bulk AdS supergravities induce the local transformations of the boundary fields $\phi_{0 I}$, which coincide with those of twodimensional conformal supergravities for $p, q \leq 2$. In particular, Weyl and super Weyl transformations on the boundary are generated from general coordinate and super transformations in the bulk.

The purpose of this paper is to compute anomalies of the local transformations of conformal supergravities on the boundary by using the AdS/CFT correspondence. By eq. (1.1) the effective action for CFTs in the background conformal supergravity fields $\phi_{0 I}$ is given by the classical supergravity action evaluated at solutions of field equations with boundary conditions $\phi_{I}=\phi_{0 I}$. Anomalies are obtained by computing variations of the supergravity action for the local symmetry transformations

$$
A=\delta S[\phi] .
$$


Although anomalies arise as a quantum effect in the CFT side, they can be obtained by classical calculations in the supergravity side. Weyl anomaly in a purely gravitational background was computed by using the AdS/CFT correspondence in ref. [8]. Our work is a generalization of this result to the case of a supergravity background. Anomalies of bosonic symmetries in the AdS/CFT correspondence were also discussed in refs. [9, 10, 11, 12, 13.

We use the three-dimensional $N=(4,4)$ AdS supergravity based on the superalgebra $\mathrm{SU}(1,1 \mid 2) \times \mathrm{SU}(1,1 \mid 2)$ to compute anomalies. As in ref. [6] the local symmetry transformations in this bulk theory will be shown to generate those of the two-dimensional $N=(4,4)$ conformal supergravity [14 on the boundary. The $N=(4,4)$ AdS supergravity arises from the type IIB supergravity on $\operatorname{AdS}_{3} \times \mathrm{S}^{3} \times \mathrm{M}_{4}$, where $\mathrm{M}_{4}$ is $\mathrm{K} 3$ or $\mathrm{T}^{4}$. This spacetime corresponds to a system of parallel $Q_{1} \mathrm{D} 1$ branes and $Q_{5}$ D5-branes in the low energy limit [15, 16]. The $Q_{1}$ D1-strings are stretching in one dimension on the $Q_{5}$ D5-branes and the remaining four-dimensional directions of the D5-branes are wrapping on $\mathrm{M}_{4}$. In the infrared limit the gauge theory on the branes is described by a $N=(4,4)$ super CFT with the central charge $c=6 Q_{1} Q_{5}=\frac{3 l}{2 G}$ [15, 16, where $l$ is the radius of the AdS space and $G$ is the threedimensional gravitational constant. The $N=(4,4)$ AdS supergravity is expected to be dual to this CFT [1, 17, 18].

We find that Weyl, axial gauge and super Weyl transformations are anomalous, while general coordinate, local Lorentz, vector gauge and local super transformations are not. The coefficients of the anomalies show that the two-dimensional CFT has the $N=(4,4)$ super Virasoro algebra with the central charge $c=\frac{3 l}{2 G}$ as expected in ref. [1], 17, 18]. This also agrees with the value of the central charge obtained in ref. [19] by computing the asymptotic symmetry algebra of three-dimensional AdS. Since our calculations of anomalies do not much depend on special properties in three dimensions, generalization to higher dimensions will be straightforward.

In the next section we give the action and the local symmetry transformation laws of the $N=(4,4)$ AdS supergravity in three dimensions. The relation to the $N=(4,4)$ conformal supergravity on the two-dimensional boundary is discussed in sect. 3. Boundary terms and counterterms to be added to the bulk action are obtained in sect. 4 and anomalies are computed in the final section. 


\section{Three-dimensional AdS supergravity}

We consider a three-dimensional AdS supergravity which has 16 supersymmetries. It is based on the superalgebra $\mathrm{SU}(1,1 \mid 2) \times \mathrm{SU}(1,1 \mid 2)$, which contains $\mathrm{SO}(2,2) \times$ $\mathrm{SU}(2) \times \mathrm{SU}(2)$ as a bosonic subalgebra. We call this theory as $N=(4,4) \operatorname{AdS}$ supergravity. Note that this theory is different from the $N=(4,4)$ theory in ref. [7] based on the superalgebra $\mathrm{OSp}(4 \mid 2) \times \mathrm{OSp}(4 \mid 2)$, which contains $\mathrm{SO}(2,2) \times \mathrm{SO}(4)$ $\times \mathrm{SO}(4)$ as a bosonic subalgebra.

The field content of this theory is a dreibein $e_{M}{ }^{A}$, Rarita-Schwinger fields $\psi_{M}^{\alpha \dot{\alpha}}$, $\psi_{M}^{\alpha^{\prime} \alpha^{\prime}}$ and $\mathrm{SU}(2) \times \mathrm{SU}(2)$ Chern-Simons gauge fields $A_{M \beta}^{\alpha}=\frac{1}{2} i\left(\sigma^{i}\right)^{\alpha}{ }_{\beta} A_{M}^{i}, A_{M \beta^{\prime}}^{\alpha^{\prime}}=$ $\frac{1}{2} i\left(\sigma^{i^{\prime}}\right)^{\alpha^{\prime}}{ }_{\beta^{\prime}} A_{M}^{i^{\prime}}$, where $\sigma^{i}, \sigma^{i^{\prime}}$ are $2 \times 2$ Pauli matrices. The undotted indices $\alpha, \beta, \cdots=$ $1,2, \alpha^{\prime}, \beta^{\prime}, \cdots=1,2$ and $i, j, \cdots=1,2,3, i^{\prime}, j^{\prime}, \cdots=1,2,3$ are those of the local $\mathrm{SU}(2) \times \mathrm{SU}(2)$ symmetry. The dotted indices $\dot{\alpha}, \dot{\beta}, \cdots=1,2$ and $\dot{\alpha}^{\prime}, \dot{\beta}^{\prime}, \cdots 1,2$ are those of the rigid $\mathrm{SU}(2) \times \mathrm{SU}(2)$ symmetry, which is not contained in $\mathrm{SU}(1,1 \mid 2) \times$ $\mathrm{SU}(1,1 \mid 2)$. We denote three-dimensional world indices as $M, N, \cdots=0,1,2$ and local Lorentz indices as $A, B, \cdots=0,1,2$. The Rarita-Schwinger fields satisfy doublysymplectic Majorana conditions

$$
\left(\psi^{\alpha \dot{\alpha}}\right)^{c}=\epsilon_{\alpha \beta} \epsilon_{\dot{\alpha} \dot{\beta}} \psi^{\beta \dot{\beta}}, \quad\left(\psi^{\alpha^{\prime} \dot{\alpha}^{\prime}}\right)^{c}=\epsilon_{\alpha^{\prime} \beta^{\prime}} \epsilon_{\dot{\alpha}^{\prime} \dot{\beta}^{\prime}} \psi^{\beta^{\prime} \dot{\beta}^{\prime}}
$$

where the superscript $c$ denotes the charge conjugation and $\epsilon_{12}=-\epsilon_{21}=-1$. We use the following conventions. The flat metric is $\eta_{A B}=\operatorname{diag}(-1,+1,+1)$ and the totally antisymmetric tensor $\epsilon^{A B C}$ is chosen as $\epsilon^{012}=+1.2 \times 2$ gamma matrices $\gamma_{A}$ satisfy $\left\{\gamma_{A}, \gamma_{B}\right\}=2 \eta_{A B}$. $\gamma^{\prime}$ 's with multiple indices are antisymmetrized products of gamma matrices with unit strength. In particular, we have $\gamma^{A B C}=-\epsilon^{A B C}$ in three dimensions. The Dirac conjugate of a spinor $\psi$ is defined as $\bar{\psi}=\psi^{\dagger} i \gamma^{0}$.

The action consists of three parts:

$$
S=S_{\text {bulk }}+S_{\text {boundary }}+\Delta S,
$$

where $S_{\text {bulk }}$ is the bulk action while $S_{\text {boundary }}$ and $\Delta S$ represent boundary terms and counterterms respectively, which will be discussed in sect. 4 . The bulk action is given by

$$
S_{\mathrm{bulk}}=\frac{1}{8 \pi G} \int d^{3} x\left[\frac{1}{2} e R+4 m^{2} e\right.
$$




$$
\begin{aligned}
& +\frac{1}{2} i \epsilon^{M N P} \bar{\psi}_{M \alpha \dot{\alpha}} \mathcal{D}_{N} \psi_{P}^{\alpha \dot{\alpha}}+\frac{1}{2} i m e \bar{\psi}_{M \alpha \dot{\alpha}} \gamma^{M N} \psi_{N}^{\alpha \dot{\alpha}} \\
& +\frac{1}{2} i \epsilon^{M N P} \bar{\psi}_{M \alpha^{\prime} \dot{\alpha}^{\prime}} \mathcal{D}_{N} \psi_{P}^{\alpha^{\prime} \dot{\alpha}^{\prime}}-\frac{1}{2} i m e \bar{\psi}_{M \alpha^{\prime} \dot{\alpha}^{\prime}} \gamma^{M N} \psi_{N}^{\alpha^{\prime} \dot{\alpha}^{\prime}} \\
& -\frac{1}{4 m} \epsilon^{M N P} \operatorname{tr}\left(A_{M} \partial_{N} A_{P}+\frac{2}{3} A_{M} A_{N} A_{P}\right) \\
& \left.+\frac{1}{4 m} \epsilon^{M N P} \operatorname{tr}\left(A_{M}^{\prime} \partial_{N} A_{P}^{\prime}+\frac{2}{3} A_{M}^{\prime} A_{N}^{\prime} A_{P}^{\prime}\right)\right],
\end{aligned}
$$

where $m$ is a positive constant and is related to the radius of the AdS space $l$ as $l=\frac{1}{2 m}$. In the following the gravitational constant will be chosen as $8 \pi G=1$. Our conventions for the curvature tensors and the covariant derivatives are

$$
\begin{aligned}
R & =e_{A}{ }^{M} e_{B}{ }^{N} R_{M N}{ }^{A B} \\
R_{M N}{ }^{A B} & =\partial_{M} \omega_{N}{ }^{A B}+\omega_{M}{ }^{A}{ }_{C} \omega_{N}{ }^{C B}-(M \leftrightarrow N), \\
\mathcal{D}_{M} \psi_{N}^{\alpha \dot{\alpha}} & =\left(\partial_{M}+\frac{1}{4} \omega_{M}{ }^{A B} \gamma_{A B}\right) \psi_{N}^{\alpha \dot{\alpha}}+A_{M \beta}^{\alpha} \psi_{N}^{\beta \dot{\alpha}}, \\
\mathcal{D}_{M} \psi_{N}^{\alpha^{\prime} \dot{\alpha}^{\prime}} & =\left(\partial_{M}+\frac{1}{4} \omega_{M}{ }^{A B} \gamma_{A B}\right) \psi_{N}^{\alpha^{\prime} \dot{\alpha}^{\prime}}+A_{M \beta^{\prime}}^{\alpha^{\prime}} \psi_{N}^{\beta^{\prime} \dot{\alpha}^{\prime}} .
\end{aligned}
$$

The covariant derivatives without $\mathrm{SU}(2) \times \mathrm{SU}(2)$ connection terms are denoted as $D_{M}$. The spin connection $\omega_{M}^{A B}$ is determined by the torsion condition

$$
D_{M} e_{N}{ }^{A}-D_{N} e_{M}{ }^{A}=\frac{1}{2} i\left(\bar{\psi}_{M \alpha \dot{\alpha}} \gamma^{A} \psi_{N}^{\alpha \dot{\alpha}}+\bar{\psi}_{M \alpha^{\prime} \dot{\alpha}^{\prime}} \gamma^{A} \psi_{N}^{\alpha^{\prime} \dot{\alpha}^{\prime}}\right)
$$

The bulk action (2.5) is invariant under the following local transformations up to total derivative terms:

$$
\begin{aligned}
\delta e_{M}{ }^{A}= & \xi^{N} \partial_{N} e_{M}{ }^{A}+\partial_{M} \xi^{N} e_{N}{ }^{A}-\lambda^{A}{ }_{B} e_{M}{ }^{B}+\frac{1}{2} i\left(\bar{\epsilon}_{\alpha \dot{\alpha}} \gamma^{A} \psi_{M}^{\alpha \dot{\alpha}}+\bar{\epsilon}_{\alpha^{\prime} \dot{\alpha}^{\prime}} \gamma^{A} \psi_{M}^{\alpha^{\prime} \dot{\alpha}^{\prime}}\right) \\
\delta \psi_{M}^{\alpha \dot{\alpha}}= & \xi^{N} \partial_{N} \psi_{M}^{\alpha \dot{\alpha}}+\partial_{M} \xi^{N} \psi_{N}^{\alpha \dot{\alpha}}-\frac{1}{4} \lambda^{A B} \gamma_{A B} \psi_{M}^{\alpha \dot{\alpha}}-\theta^{\alpha}{ }_{\beta} \psi_{M}^{\beta \dot{\alpha}}+\mathcal{D}_{M} \epsilon^{\alpha \dot{\alpha}}+m \gamma_{M} \epsilon^{\alpha \dot{\alpha}} \\
\delta \psi_{M}^{\alpha^{\prime} \dot{\alpha}^{\prime}}= & \xi^{N} \partial_{N} \psi_{M}^{\alpha^{\prime} \dot{\alpha}^{\prime}}+\partial_{M} \xi^{N} \psi_{N}^{\alpha^{\prime} \dot{\alpha}^{\prime}}-\frac{1}{4} \lambda^{A B} \gamma_{A B} \psi_{M}^{\alpha^{\prime} \dot{\alpha}^{\prime}}-\theta^{\alpha^{\prime}}{ }_{\beta^{\prime}} \psi_{M}^{\beta^{\prime} \dot{\alpha}^{\prime}} \\
& +\mathcal{D}_{M} \epsilon^{\alpha^{\prime} \dot{\alpha}^{\prime}}-m \gamma_{M} \epsilon^{\alpha^{\prime} \dot{\alpha}^{\prime}} \\
\delta A_{M}^{i}= & \xi^{N} \partial_{N} A_{M}^{i}+\partial_{M} \xi^{N} A_{N}^{i}+\mathcal{D}_{M} \theta^{i}-2 m \bar{\epsilon}_{\alpha \dot{\alpha}} \psi_{M}^{\beta \dot{\alpha}}\left(\sigma^{i}\right)^{\alpha}{ }_{\beta}, \\
\delta A_{M}^{i^{\prime}}= & \xi^{N} \partial_{N} A_{M}^{i^{\prime}}+\partial_{M} \xi^{N} A_{N}^{i^{\prime}}+\mathcal{D}_{M} \theta^{i^{\prime}}+2 m \bar{\epsilon}_{\alpha^{\prime} \dot{\alpha}^{\prime}} \psi_{M}^{\beta^{\prime} \dot{\alpha}^{\prime}}\left(\sigma^{i^{\prime}}\right)^{\alpha^{\prime}}{ }_{\beta^{\prime}} .
\end{aligned}
$$

The transformation parameters $\xi^{M}(x), \lambda^{A B}(x), \theta^{\alpha}{ }_{\beta}(x)=\frac{1}{2} i\left(\sigma^{i}\right)^{\alpha}{ }_{\beta} \theta^{i}(x), \theta^{\alpha^{\prime}}{ }_{\beta^{\prime}}(x)=$ $\frac{1}{2} i\left(\sigma^{i^{\prime}}\right)^{\alpha^{\prime}}{ }_{\beta^{\prime}} \theta^{i^{\prime}}(x)$, and $\epsilon^{\alpha \dot{\alpha}}(x), \epsilon^{\alpha^{\prime} \dot{\alpha}^{\prime}}(x)$ represent general coordinate $\left(\delta_{G}\right)$, local Lorentz 
$\left(\delta_{L}\right)$, $\mathrm{SU}(2) \times \mathrm{SU}(2)$ gauge $\left(\delta_{g}\right)$ and local super $\left(\delta_{Q}\right)$ transformations respectively. The parameters $\epsilon^{\alpha \dot{\alpha}}, \epsilon^{\alpha^{\prime} \dot{\alpha}^{\prime}}$ satisfy the doubly-symplectic Majorana conditions (2.3). The commutator algebra of these transformations closes modulo the field equations. In particular, the commutator of two local supertransformations with parameters $\epsilon_{1}$ and $\epsilon_{2}$ is

$$
\left[\delta_{Q}\left(\epsilon_{1}\right), \delta_{Q}\left(\epsilon_{2}\right)\right]=\delta_{G}(\xi)+\delta_{L}(\lambda)+\delta_{g}(\theta)+\delta_{Q}(\epsilon),
$$

where

$$
\begin{aligned}
& \xi^{M}=\frac{1}{2} i\left(\bar{\epsilon}_{2 \alpha \dot{\alpha}} \gamma^{M} \epsilon_{1}^{\alpha \dot{\alpha}}+\bar{\epsilon}_{2 \alpha^{\prime} \dot{\alpha}^{\prime}} \gamma^{M} \epsilon_{1}^{\alpha^{\prime} \dot{\alpha}^{\prime}}\right), \\
& \lambda^{A B}=-\xi^{M} \omega_{M}^{A B}-i m\left(\bar{\epsilon}_{2 \alpha \dot{\alpha}} \gamma^{A B} \epsilon_{1}^{\alpha \dot{\alpha}}-\bar{\epsilon}_{2 \alpha^{\prime} \dot{\alpha}^{\prime}} \gamma^{A B} \epsilon_{1}^{\alpha^{\prime} \dot{\alpha}^{\prime}}\right) \text {, } \\
& \theta^{i}=-\xi^{M} A_{M}^{i}-2 m \bar{\epsilon}_{2 \alpha \dot{\gamma}} \epsilon_{1}^{\beta \dot{\gamma}}\left(\sigma^{i}\right)^{\alpha}{ }_{\beta}, \\
& \theta^{i^{\prime}}=-\xi^{M} A_{M}^{i^{\prime}}+2 m \bar{\epsilon}_{2 \alpha^{\prime} \dot{\gamma}^{\prime}} \epsilon_{1}^{\beta^{\prime} \dot{\gamma}^{\prime}}\left(\sigma^{i^{\prime}}\right)^{\alpha^{\prime}{ }_{\beta^{\prime}},} \\
& \epsilon^{\alpha \dot{\alpha}}=-\xi^{M} \psi_{M}^{\alpha \dot{\alpha}}, \quad \epsilon^{\alpha^{\prime} \dot{\alpha}^{\prime}}=-\xi^{M} \psi_{M}^{\alpha^{\prime} \dot{\alpha}^{\prime}} .
\end{aligned}
$$

The action (2.5) is also invariant under the rigid $\mathrm{SU}(2) \times \mathrm{SU}(2)$ transformations, which act on the dotted indices of $\psi_{M}^{\alpha \dot{\alpha}}, \psi_{M}^{\alpha^{\prime} \dot{\alpha}^{\prime}}$.

Three-dimensional AdS supergravities with $N=(p, q)$ supersymmetries for $p, q=1,2,4$ can be obtained from the above $N=(4,4)$ theory by consistent truncations. For instance, the $N=(2,2)$ theory in ref. [7] can be obtained by a truncation

$$
\psi^{1 \dot{2}}=\psi^{2 \dot{1}}=\psi^{1^{\prime} \dot{2}^{\prime}}=\psi^{2^{\prime} \mathrm{i}^{\prime}}=0, \quad A_{M}^{1}=A_{M}^{2}=A_{M}^{1^{\prime}}=A_{M}^{2^{\prime}}=0 .
$$

Redefining the remaining fields as

$$
\psi_{M}^{1} \equiv \frac{1}{\sqrt{2}}\left(\psi_{M}^{11}+\psi_{M}^{2 \dot{2}}\right), \quad \psi_{M}^{2} \equiv \frac{1}{\sqrt{2}} i\left(\psi_{M}^{1 \dot{1}}-\psi_{M}^{2 \dot{2}}\right), \quad A_{M}^{12}=-A_{M}^{21} \equiv \frac{1}{2} A_{M}^{3}
$$

and similarly for $\psi_{M}^{1^{\prime}}, \psi_{M}^{2^{\prime}}, A_{M}^{1^{\prime} 2^{\prime}}$, we obtain the action and the local transformations of the $N=(2,2)$ theory [7]. $A_{M}^{12}, A_{M}^{1^{\prime} 2^{\prime}}$ are the $\mathrm{SO}(2) \times \mathrm{SO}(2)$ gauge fields and $\psi_{M}^{i}$, $\psi_{M}^{i^{\prime}}\left(i, i^{\prime}=1,2\right)$ are Majorana spinors. The $N=(1,1)$ theory [7] is obtained by further truncation

$$
\psi_{M}^{2}=\psi_{M}^{2^{\prime}}=0, \quad A_{M}^{12}=A_{M}^{1^{\prime 22^{\prime}}}=0 .
$$

The $(p, q)$ theories for other values of $p, q$ can be also obtained by appropriate truncations. 


\section{Local symmetries on the boundary}

As in ref. [6] we partially fix the gauge for the local symmetries (2.8) and obtain how the residual local symmetries act on the fields on the boundary. We represent the three-dimensional AdS space as a region $x^{2}>0$ in $\mathbf{R}^{3}$ with coordinates $\left(x^{0}, x^{1}, x^{2}\right)$. The boundary of the AdS space corresponds to a plane $x^{2}=0$ and a point $x^{2}=\infty$. Our gauge fixing conditions are

$$
\begin{aligned}
& e_{M=2}^{A=2}=\frac{1}{2 m x^{2}}, \quad e_{M=2}{ }^{a}=0, \quad e_{\mu}^{A=2}=0, \\
& \psi_{2}^{\alpha \dot{\alpha}}=0, \quad \psi_{2}^{\alpha^{\prime} \dot{\alpha}^{\prime}}=0, \quad A_{2 \beta}^{\alpha}=0, \quad A_{2}^{\alpha^{\prime}{ }^{\prime}}=0,
\end{aligned}
$$

where $\mu, \nu, \cdots=0,1$ and $a, b, \cdots=0,1$ are two-dimensional world indices and local Lorentz indices respectively. The metric in this gauge has a form

$$
d x^{M} d x^{N} g_{M N}=\frac{1}{\left(2 m x^{2}\right)^{2}}\left(d x^{2} d x^{2}+d x^{\mu} d x^{\nu} \hat{g}_{\mu \nu}\right)
$$

The $\mathrm{SO}(2,2)$ invariant AdS metric corresponds to the case $\hat{g}_{\mu \nu}=\eta_{\mu \nu}$ but we consider the general $\hat{g}_{\mu \nu}$. We define $\hat{e}_{\mu}{ }^{a}$ by $\hat{g}_{\mu \nu}=\hat{e}_{\mu}{ }^{a} \hat{e}_{\nu}{ }^{b} \eta_{a b}$ and

$$
\psi_{\mu \pm}^{\alpha \dot{\alpha}}=\left(2 m x^{2}\right)^{\mp \frac{1}{2}} \hat{\psi}_{\mu \pm}^{\alpha \dot{\alpha}}, \quad \psi_{\mu \pm}^{\alpha^{\prime} \dot{\alpha}^{\prime}}=\left(2 m x^{2}\right)^{ \pm \frac{1}{2}} \hat{\psi}_{\mu \pm}^{\alpha^{\prime} \dot{\alpha}^{\prime}}, \quad A_{\mu \beta}^{\alpha}=\hat{A}_{\mu \beta}^{\alpha}, \quad A_{\mu \beta^{\prime}}^{\alpha^{\prime}}=\hat{A}_{\mu \beta^{\prime}}^{\alpha^{\prime}}
$$

where $\psi_{\mu \pm}=\frac{1}{2}\left(1 \pm \gamma^{2}\right) \psi_{\mu}$. As discussed in ref. [6] the field equations show that the fields with ^ behave as $\mathcal{O}\left(\left(x^{2}\right)^{0}\right)$ near the boundary. We impose boundary conditions on the fields at $x^{2}=0$ as

$$
\begin{aligned}
& \hat{e}_{\mu}{ }^{a}=e_{0 \mu}{ }^{a}, \quad \hat{\psi}_{\mu+}^{\alpha \dot{\alpha}}=\psi_{0 \mu+}^{\alpha \dot{\alpha}}, \quad \hat{\psi}_{\mu-}^{\alpha^{\prime} \dot{\alpha}^{\prime}}=\psi_{0 \mu-}^{\alpha^{\prime} \dot{\alpha}^{\prime}}, \\
& \hat{A}_{\mu}^{(+) \alpha}{ }_{\beta}=A_{0 \mu}^{(+) \alpha}{ }_{\beta}, \quad \hat{A}_{\mu}^{(-) \alpha^{\prime}}{ }_{\beta^{\prime}}=A_{0 \mu}^{(-) \alpha^{\prime}}{ }_{\beta^{\prime}},
\end{aligned}
$$

where $\hat{A}_{\mu}^{( \pm)}=\frac{1}{2}\left(\hat{g}_{\mu \nu} \pm \hat{e} \epsilon_{\mu \nu}\right) \hat{A}^{\nu}$. The fields $\phi_{0 I}=\left(e_{0 \mu}{ }^{a}, \psi_{0 \mu+}^{\alpha \dot{\alpha}}, \psi_{0 \mu-}^{\alpha^{\prime} \dot{\alpha}^{\prime}}, A_{0 \mu}^{(+) \alpha}{ }_{\beta}, A_{0 \mu}^{(-) \alpha^{\prime}{ }^{\prime}}\right)$ are fixed functions on the boundary. Since field equations of the Rarita-Schwinger fields and the gauge fields are first order, boundary conditions are imposed on only half of their components. Other components of the fields on the boundary are nonlocal functionals of the fields $\phi_{0 I}$, which are obtained by solving the field equations. We also introduce notations $\psi_{0 \mu-}^{\alpha \dot{\alpha}}, \psi_{0 \mu+}^{\alpha^{\prime} \dot{\alpha}^{\prime}}, A_{0 \mu}^{(-) \alpha}{ }_{\beta}, A_{0 \mu}^{(+) \alpha^{\prime}}{ }_{\beta^{\prime}}$ defined by $\hat{\psi}_{\mu-}^{\alpha \dot{\alpha}} \rightarrow \psi_{0 \mu-}^{\alpha \dot{\alpha}}$, etc. 
The parameters of the residual symmetries, which preserve the gauge conditions (3.14), near the boundary $x^{2}=0$ are

$$
\begin{aligned}
\xi^{2} & =-x^{2} \Lambda_{0}\left(x^{0}, x^{1}\right), \quad \xi^{\mu}=\xi_{0}^{\mu}\left(x^{0}, x^{1}\right)+\mathcal{O}\left(\left(x^{2}\right)^{2}\right), \\
\lambda^{a b} & =\lambda_{0}^{a b}\left(x^{0}, x^{1}\right)+\mathcal{O}\left(x^{2}\right), \quad \lambda^{a 2}=\mathcal{O}\left(x^{2}\right), \\
\epsilon_{ \pm}^{\alpha \dot{\alpha}} & =\left(2 m x^{2}\right)^{\mp \frac{1}{2}}\left[\epsilon_{0 \pm}^{\alpha \dot{\alpha}}\left(x^{0}, x^{1}\right)+\mathcal{O}\left(x^{2}\right)\right], \\
\epsilon_{ \pm}^{\alpha^{\prime} \dot{\alpha}^{\prime}} & =\left(2 m x^{2}\right)^{ \pm \frac{1}{2}}\left[\epsilon_{0 \pm}^{\alpha^{\prime} \dot{\alpha}^{\prime}}\left(x^{0}, x^{1}\right)+\mathcal{O}\left(x^{2}\right)\right], \\
\theta^{\alpha}{ }_{\beta} & =\theta_{0 \beta}^{\alpha}\left(x^{0}, x^{1}\right)+\mathcal{O}\left(\left(x^{2}\right)^{2}\right), \quad \theta_{\beta^{\prime}}^{\alpha^{\prime}}=\theta_{0}^{\alpha^{\prime}}{ }_{\beta^{\prime}}\left(x^{0}, x^{1}\right)+\mathcal{O}\left(\left(x^{2}\right)^{2}\right),
\end{aligned}
$$

where $\Lambda_{0}, \xi_{0}^{\mu}, \lambda_{0}^{a b}, \epsilon_{0 \pm}^{\alpha \dot{\alpha}}, \epsilon_{0 \pm}^{\alpha^{\prime} \dot{\alpha}^{\prime}}, \theta_{0}^{\alpha}$ and $\theta_{0}^{\alpha^{\prime}} \beta^{\prime}$ are arbitrary functions of $x^{0}$ and $x^{1}$. Order $\mathcal{O}\left(x^{2}\right)$ and $\mathcal{O}\left(\left(x^{2}\right)^{2}\right)$ terms are non-local functionals of these functions and the fields $\phi_{0 I}$. Substituting eqs. (3.16), (3.17), (3.18) into eq. (2.8) and taking the limit $x^{2} \rightarrow 0$ we obtain transformations of the fields on the boundary $\phi_{0 I}$. These transformations are shown to coincide with those of the two-dimensional $(4,4)$ conformal supergravity |14.

The two-dimensional $(4,4)$ conformal supergravity [14] contains a zweibein $\tilde{e}_{\mu}{ }^{a}$, doubly-symplectic Majorana Rarita-Schwinger fields $\tilde{\psi}_{\mu}^{\alpha \dot{\alpha}}$ and $\mathrm{SU}(2)$ gauge fields $\tilde{A}_{\mu \beta}^{\alpha}$. These fields are identified with the boundary fields $\phi_{0 I}$ as

$$
\tilde{e}_{\mu}{ }^{a}=e_{0 \mu}{ }^{a}, \quad \tilde{\psi}_{\mu}^{\alpha \dot{\alpha}}=\psi_{0 \mu+}^{\alpha \dot{\alpha}}+\psi_{0 \mu-}^{\alpha^{\prime} \dot{\alpha}^{\prime}}, \quad \tilde{A}_{\mu \beta}^{\alpha}=A_{0 \mu}^{(+) \alpha}{ }_{\beta}+A_{0 \mu}^{(-) \alpha^{\prime}}{ }_{\beta^{\prime}},
$$

where $\alpha^{\prime}=\alpha, \dot{\alpha}^{\prime}=\dot{\alpha}, \beta^{\prime}=\beta$. We find that transformation laws of these fields derived from the three-dimensional transformations (2.8) are

$$
\begin{aligned}
\delta \tilde{e}_{\mu}{ }^{a}= & \tilde{\xi}^{\nu} \partial_{\nu} \tilde{e}_{\mu}{ }^{a}+\partial_{\mu} \tilde{\xi}^{\nu} \tilde{e}_{\nu}{ }^{a}+\tilde{\Lambda} e_{\mu}{ }^{a}-\tilde{\lambda}^{a}{ }_{b} \tilde{e}_{\mu}{ }^{b}+\frac{1}{2} i \overline{\tilde{\epsilon}}_{\alpha \dot{\alpha}} \gamma^{a} \tilde{\psi}_{\mu}^{\alpha \dot{\alpha}} \\
\delta \tilde{\psi}_{\mu}^{\alpha \dot{\alpha}}= & \tilde{\xi}^{\nu} \partial_{\nu} \tilde{\psi}_{\mu}^{\alpha \dot{\alpha}}+\partial_{\mu} \tilde{\xi}^{\nu} \tilde{\psi}_{\nu}^{\alpha \dot{\alpha}}+\frac{1}{2} \tilde{\Lambda}_{\bar{\psi}}^{\alpha \dot{\alpha}}-\frac{1}{4} \tilde{\lambda}^{a b} \gamma_{a b} \tilde{\psi}_{\mu}^{\alpha \dot{\alpha}} \\
& -\tilde{\theta}_{\mathrm{V} \beta}^{\alpha} \tilde{\psi}_{\mu}^{\beta \dot{\alpha}}-\tilde{\theta}_{\mathrm{A} \beta}^{\alpha} \gamma_{2} \tilde{\psi}_{\mu}^{\beta \dot{\alpha}}+\tilde{\mathcal{D}}_{\mu} \tilde{\epsilon}^{\alpha \dot{\alpha}}+\tilde{\gamma}_{\mu} \tilde{\eta}^{\alpha \dot{\alpha}} \\
\delta \tilde{A}_{\mu}^{i}= & \tilde{\xi}^{\nu} \partial_{\nu} A_{\mu}^{i}+\partial_{\mu} \tilde{\xi}^{\nu} \tilde{A}_{\nu}^{i}+\tilde{\mathcal{D}}_{\mu} \tilde{\theta}_{\mathrm{V}}^{i}+\tilde{e} \epsilon_{\mu \nu} \tilde{\mathcal{D}}^{\nu} \tilde{\theta}_{\mathrm{A}}^{i} \\
& +\left(\frac{1}{4} \overline{\tilde{\epsilon}}_{\alpha \dot{\alpha}} \tilde{\gamma}_{\mu} \tilde{\gamma}^{\rho \sigma} \tilde{\psi}_{\rho \sigma}^{\beta \dot{\alpha}}+\frac{1}{2} \overline{\tilde{\eta}}_{\alpha \dot{\alpha}} \tilde{\gamma}^{\nu} \tilde{\gamma}_{\mu} \tilde{\psi}_{\nu}^{\beta \dot{\alpha}}\right)\left(\sigma^{i}\right)^{\alpha}{ }_{\beta},
\end{aligned}
$$

where $\tilde{\mathcal{D}}_{\mu}$ is the covariant derivative including the gauge field $\tilde{A}_{\mu \beta}^{\alpha}$ and $\tilde{\psi}_{\mu \nu}^{\alpha \dot{\alpha}}$ is the field strengths of the Rarita-Schwinger fields

$$
\begin{aligned}
\tilde{\mathcal{D}}_{\mu} \tilde{\epsilon}^{\alpha \dot{\alpha}} & =\tilde{D}_{\mu} \tilde{\epsilon}^{\alpha \dot{\alpha}}+\tilde{A}_{\mu \beta}^{\alpha} \tilde{\epsilon}^{\beta \dot{\alpha}} \\
\tilde{\psi}_{\mu \nu}^{\alpha \dot{\alpha}} & =\tilde{\mathcal{D}}_{\mu} \tilde{\psi}_{\nu}^{\alpha \dot{\alpha}}-\tilde{\mathcal{D}}_{\nu} \tilde{\psi}_{\mu}^{\alpha \dot{\alpha}} .
\end{aligned}
$$


The transformation parameters with a tilde in eq. (3.20) are related to those in eq. (3.18) as

$$
\begin{aligned}
\tilde{\xi}^{\mu} & =\xi^{\mu}, \quad \tilde{\Lambda}=\Lambda_{0}, \quad \tilde{\lambda}^{a b}=\lambda_{0}^{a b}, \quad \tilde{\epsilon}^{\alpha \dot{\alpha}}=\epsilon_{0+}^{\alpha \dot{\alpha}}+\epsilon_{0-}^{\alpha^{\prime} \dot{\alpha}^{\prime}}, \\
\tilde{\eta}^{\alpha \dot{\alpha}} & =2 m\left(\epsilon_{0-}^{\alpha \dot{\alpha}}-\epsilon_{0+}^{\alpha^{\prime} \dot{\alpha}^{\prime}}\right)+\frac{1}{2}\left(A_{0 \beta}^{\alpha}-A_{0}^{\alpha^{\prime}{ }^{\prime}}\right) \gamma_{2} \tilde{\epsilon}^{\beta \dot{\alpha}}, \\
\tilde{\theta}_{\mathrm{V} \beta}^{\alpha} & =\frac{1}{2}\left(\theta_{0 \beta}^{\alpha}+\theta_{0}^{\alpha^{\prime}}{ }^{\prime}\right), \quad \tilde{\theta}_{\mathrm{A} \beta}^{\alpha}=\frac{1}{2}\left(\theta_{0 \beta}^{\alpha}-\theta_{0}^{\alpha^{\prime}}{ }^{\prime}\right),
\end{aligned}
$$

where $\alpha^{\prime}=\alpha, \dot{\alpha}^{\prime}=\dot{\alpha}, \beta^{\prime}=\beta$. The transformations (3.20) represent general coordinate $\left(\tilde{\xi}^{\mu}\right)$, local Lorentz $\left(\tilde{\lambda}^{a b}\right)$, Weyl $(\tilde{\Lambda})$, vector $\operatorname{SU}(2)$ gauge $\left(\theta_{\mathrm{V} \beta}^{\alpha}\right)$, axial vector gauge $\left(\theta_{\mathrm{A} \beta}^{\alpha}\right)$, local super $\left(\tilde{\epsilon}^{\alpha \dot{\alpha}}\right)$ and super Weyl $\left(\tilde{\eta}^{\alpha \dot{\alpha}}\right)$ transformations. These are local symmetry transformations of the $(4,4)$ conformal supergravity [14.

\section{Boundary terms and counterterms}

To compute anomalies (1.2) for the local transformations (3.20) we need the total action (2.4) including the boundary terms and the local counterterms. The boundary terms are chosen such that the variational principle is well-defined [20, 21, 22]. When we take a variation of the action, we obtain boundary terms arising from partial integrations in addition to bulk terms proportional to field equations. Such boundary terms should be cancelled by a variation of $S_{\text {boundary }}$ in eq. (2.4) when the boundary conditions (3.17) are used. We find that appropriate boundary terms are

$$
\begin{aligned}
S_{\text {boundary }}= & \int d^{2} x\left[\frac{1}{2} \epsilon^{\mu \nu} \epsilon_{A B C} e_{\mu}{ }^{A} \omega_{\nu}^{B C}-\frac{1}{2} i \epsilon^{\mu \nu}\left(\bar{\psi}_{\mu+\alpha \dot{\alpha}} \psi_{\nu-}^{\alpha \dot{\alpha}}+\bar{\psi}_{\mu-\alpha^{\prime} \dot{\alpha}^{\prime}} \psi_{\nu+}^{\alpha^{\prime} \dot{\alpha}^{\prime}}\right)\right. \\
& \left.-\frac{1}{8 m} \hat{e} \hat{g}^{\mu \nu} \operatorname{tr}\left(A_{\mu} A_{\nu}+A_{\mu}^{\prime} A_{\nu}^{\prime}\right)\right] .
\end{aligned}
$$

The first term is equivalent to the usual boundary term for the Einstein action, which is proportional to the trace of the second fundamental form of the boundary 23.

The bulk action and the boundary terms are both divergent. One has to regularize them and subtract singular terms by the counterterms $\Delta S$. The singular terms turn out to be local functionals of the fields $\phi_{0 I}$. We regularize divergences by restricting the range of $x^{2}$ to $x^{2}>\epsilon$, where $\epsilon>0$ is a cut-off parameter. The boundary is now at $x^{2}=\epsilon$. The regularization is removed for $\epsilon \rightarrow 0$. 
Let us evaluate singular terms in $S_{\text {bulk }}$ and $S_{\text {boundary }}$ to obtain the local counterterms $\Delta S$. Using the field equations the bulk action becomes

$$
S_{\text {bulk }}=\int d^{2} x \int_{\epsilon}^{\infty} d x^{2}\left[-\frac{1}{m\left(x^{2}\right)^{3}} \hat{e}-\frac{1}{4 x^{2}} i \hat{e}\left(\overline{\hat{\psi}}_{\mu \alpha \dot{\alpha}} \hat{\gamma}^{\mu \nu} \hat{\psi}_{\nu}^{\alpha \dot{\alpha}}-\overline{\hat{\psi}}_{\mu \alpha^{\prime} \dot{\alpha}^{\prime}} \hat{\gamma}^{\mu \nu} \hat{\psi}_{\nu}^{\alpha^{\prime} \dot{\alpha}^{\prime}}\right)\right]
$$

As in ref. [8] we then expand the metric in $x^{2}$ as

$$
\hat{g}_{\mu \nu}=g_{0 \mu \nu}+\left(2 m x^{2}\right)^{2} g_{(2) \mu \nu}+\left(2 m x^{2}\right)^{2} \log \left(2 m x^{2}\right)^{2} h_{(2) \mu \nu}+\mathcal{O}\left(\left(x^{2}\right)^{3}\right)
$$

Coefficients of these expansion $g_{(2) \mu \nu}, h_{(2) \mu \nu}, \cdots$ are determined as functions of the fields $\phi_{0 I}$ by solving the field equations. To compute singular terms in eq. (4.24) we need to know the expansion of $\hat{e}=\sqrt{-\hat{g}}$, which are determined by $g_{0}^{\mu \nu} g_{(2) \mu \nu}$ and $g_{0}^{\mu \nu} h_{(2) \mu \nu}$. They can be obtained from the (22) component of the Einstein equation as in ref. [8]. We find $g_{0}^{\mu \nu} h_{(2) \mu \nu}=0$ and

$$
g_{0}^{\mu \nu} g_{(2) \mu \nu}=-\frac{1}{8 m^{2}}\left[R_{0}+i m\left(\bar{\psi}_{0 \mu \alpha \dot{\alpha}} \gamma_{0}^{\mu \nu} \psi_{0 \nu}^{\alpha \dot{\alpha}}-\bar{\psi}_{0 \mu \alpha^{\prime} \dot{\alpha}^{\prime}} \gamma_{0}^{\mu \nu} \psi_{0 \nu}^{\alpha^{\prime} \dot{\alpha}^{\prime}}\right)\right]
$$

Then we find singular terms in eq. (4.24) as

$$
S_{\text {bulk }}^{\text {(div) }}=\int d^{2} x e_{0}\left[-\frac{1}{2 m \epsilon^{2}}-\frac{1}{4 m} \log \epsilon R_{0}\right]
$$

The singularities of $\psi$ terms in eq. (4.24) have been cancelled by those coming from eq. (4.26). To obtain singular terms in the boundary terms (4.23) we first note that all the terms in eq. (4.23) except the first are finite. By using the explicit form of the spin connection the first term can be written as

$$
\begin{aligned}
\int d^{2} x \frac{1}{2} \epsilon^{\mu \nu} \epsilon_{A B C} e_{\mu}{ }^{A} \omega_{\nu}^{B C} & =\int d^{2} x\left[\frac{1}{m \epsilon^{2}} \hat{e}-\frac{1}{2 m \epsilon} \partial_{2} \hat{e}\right] \\
& =\int d^{2} x \frac{1}{m \epsilon^{2}} e_{0}+\mathcal{O}(\epsilon),
\end{aligned}
$$

where we have used eqs. (4.25), (4.26) in the second equality. The counterterms are chosen to cancel the singularities in eqs. (4.27), (4.28) as

$$
\Delta S=\int d^{2} x\left[-\frac{1}{2 m \epsilon^{2}} e_{0}+\frac{1}{4 m} \log \epsilon e_{0} R_{0}\right]+\Delta S_{\text {finite }}
$$


where $\Delta S_{\text {finite }}$ is a finite local term. The second term of the integrand is proportional to the Euler density, which is a total derivative locally, and will be ignored in the following.

We note that $\Delta S$ is a local functional of the boundary fields $\phi_{0 I}$. In principle, it could depend on other components of the fields such as $\psi_{0 \mu-}^{\alpha \dot{\alpha}}$, which is a non-local functional of $\phi_{0 I}$. From the AdS point of view it is not clear why they should be local. The locality of singular terms may be understood by the IR-UV connection [24]. Infrared divergences in the bulk theory can be interpreted as ultraviolet divergences in the boundary CFT. Since ultraviolet divergences are short distance property of the CFT, they should be local.

\section{Anomalies}

We compute a variation of the total action (2.4) under the local symmetry transformations (3.20) and obtain anomalies of the two-dimensional $(4,4)$ CFT. Since the boundary transformations (3.20) are generated from the bulk transformations (2.8), we can use the latter to compute a variation in the bulk. Let us first consider the bosonic transformations. For the moment we assume $\Delta S_{\text {finite }}=0$ in eq. (4.29). Under the three-dimensional general coordinate transformation with a parameter $\xi^{M}$ the bulk action transforms as

$$
\begin{aligned}
\delta_{G} S_{\text {bulk }} & =\int d^{2} x \int_{\epsilon}^{\infty} d x^{2} \partial_{M}\left(\xi^{M} \mathcal{L}_{\text {bulk }}\right) \\
& =\int d^{2} x \epsilon \Lambda_{0} \mathcal{L}_{\text {bulk }} \\
& =\int d^{2} x e_{0} \Lambda_{0}\left[-\frac{1}{m \epsilon^{2}}+\frac{1}{4 m} R_{0}\right],
\end{aligned}
$$

where $\mathcal{L}_{\text {bulk }}$ is the integrand of eq. (2.5) or eq. (4.24). In the last equality we have used eq. (4.26). The transformation of the boundary terms (4.23) and the local counterterms (4.29) can be easily computed by using the transformation laws (3.20) and eq. (4.28). Using the quantities in eqs. (3.19), (3.22) we obtain the transformation of the total action as

$$
\delta_{G} S=\frac{1}{4 m} \int d^{2} x \tilde{e} \tilde{\Lambda} \tilde{R} .
$$


We see that the total action is invariant under the two-dimensional general coordinate transformation but not under the Weyl transformation. As for the local Lorentz transformation the bulk action (2.5) and the local counterterms (4.29) are manifestly invariant. The boundary action (4.23) is also invariant when the first term is expressed as in eq. (4.28). Therefore, the total action is invariant under the local Lorentz transformation

$$
\delta_{L} S=0
$$

Under the $\mathrm{SU}(2) \times \mathrm{SU}(2)$ gauge transformation the Chern-Simons terms in the bulk action (2.5) give two-dimensional chiral gauge anomaly [25]. Combining with the transformation of the boundary action we obtain

$$
\delta_{g} S=\frac{1}{2 m} \int d^{2} x \epsilon^{\mu \nu} \operatorname{tr}\left(\theta_{0} \partial_{\mu} A_{0 \nu}^{(+)}-\theta_{0}^{\prime} \partial_{\mu} A_{0 \nu}^{\prime(-)}\right)
$$

It is possible to preserve the diagonal vector $\mathrm{SU}(2)$ subgroup of the $\mathrm{SU}(2) \times \mathrm{SU}(2)$ gauge symmetry by introducing an appropriate finite counterterm. By choosing

$$
\Delta S_{\text {finite }}=-\frac{1}{2 m} \int d^{2} x \epsilon^{\mu \nu} \operatorname{tr}\left(A_{0 \mu}^{(+)} A_{0 \nu}^{\prime(-)}\right)
$$

we obtain

$$
\delta_{g} S=\frac{1}{2 m} \int d^{2} x \epsilon^{\mu \nu} \operatorname{tr}\left(\tilde{\theta}_{\mathrm{A}} \tilde{F}_{\mu \nu}\right)
$$

where $\tilde{F}_{\mu \nu}$ is the field strength of the $\mathrm{SU}(2)$ gauge field $\tilde{A}_{\mu}$. We see that there is no anomaly in the vector $\mathrm{SU}(2)$ gauge transformation with the parameter $\tilde{\theta}_{\mathrm{V}}$. Since the finite counterterm (5.34) is invariant under the general coordinate and local Lorentz transformations, the introduction of it does not change the results (5.31), (5.32).

To obtain the supertransformation of the action we first compute the variation of the total action as

$$
\begin{aligned}
\delta_{Q} S= & \frac{1}{2 m} \int d^{2} x\left[\frac{1}{\epsilon} \hat{e} \hat{e}_{a}{ }^{\mu} \delta_{Q} \omega_{\mu}^{a 2}+\frac{1}{\epsilon^{2}} \delta_{Q} e_{0}\right. \\
& -2 i m \epsilon^{\mu \nu}\left(\delta_{Q} \bar{\psi}_{0 \mu+\alpha \dot{\alpha}} \psi_{0 \nu-}^{\alpha \dot{\alpha}}+\delta_{Q} \bar{\psi}_{0 \mu-\alpha^{\prime} \dot{\alpha}^{\prime}} \psi_{0 \nu+}^{\alpha^{\prime} \dot{\alpha}^{\prime}}\right) \\
& -e_{0} \operatorname{tr}\left(A_{0}^{(-) \mu} \delta_{Q} A_{0 \mu}+A_{0}^{\prime(+) \mu} \delta_{Q} A_{0 \mu}^{\prime}\right) \\
& \left.-\frac{1}{4} \delta_{Q}\left(e_{0} g_{0}^{\mu \nu}\right) \operatorname{tr}\left(A_{0 \mu} A_{0 \nu}+A_{0 \mu}^{\prime} A_{0 \nu}^{\prime}\right)\right]+\delta_{Q} \Delta S_{\text {finite }} .
\end{aligned}
$$


The first two terms can be written as

$$
\begin{aligned}
& \frac{1}{\epsilon} \hat{e} \hat{e}_{a}{ }^{\mu} \delta_{Q} \omega_{\mu}{ }^{a 2}+\frac{1}{\epsilon^{2}} \delta_{Q} e_{0} \\
& \quad=-\frac{1}{\epsilon} \hat{e} \delta_{Q} \hat{e}_{a}{ }^{\mu} \bar{\omega}_{\mu}^{a 2}+\frac{1}{\epsilon} \hat{e} \delta_{Q}\left(\hat{e}^{-1} \partial_{2} \hat{e}\right)-\frac{1}{\epsilon^{2}} \delta_{Q} \hat{e}+\frac{1}{\epsilon^{2}} \delta_{Q} e_{0} \\
& \quad=-\frac{1}{\epsilon} \hat{e} \delta_{Q} \hat{e}_{a}{ }^{\mu} \bar{\omega}_{\mu}{ }^{a 2}+2 m^{2} \delta_{Q}\left(e_{0} g_{0}^{\mu \nu} g_{(2) \mu \nu}\right)-4 m^{2} \delta_{Q} e_{0} g_{0}^{\mu \nu} g_{(2) \mu \nu}
\end{aligned}
$$

where $\bar{\omega}_{\mu}^{a 2}=\omega_{\mu}^{a 2}+2 m e_{\mu}^{a}$ and we have used eq. (4.25). The second term in the last equation does not contribute because of the formula

$$
\delta_{Q} \int d^{2} x e_{0} g_{0}^{\mu \nu} g_{(2) \mu \nu}=0
$$

This can be shown by using the Rarita-Schwinger field equations expressed as

$$
\begin{aligned}
\epsilon^{\mu \nu} \mathcal{D}_{0 \mu} \psi_{0 \nu-}^{\alpha \dot{\alpha}} & =-\frac{1}{4 m \epsilon} \epsilon^{\mu \nu} \gamma_{a} \psi_{0 \nu+}^{\alpha \dot{\alpha}} \bar{\omega}_{\mu}{ }^{a 2}, \\
\epsilon^{\mu \nu} \mathcal{D}_{0 \mu} \psi_{0 \nu+}^{\alpha^{\prime} \dot{\alpha}^{\prime}} & =\frac{1}{4 m \epsilon} \epsilon^{\mu \nu} \gamma_{a} \psi_{0 \nu-}^{\alpha^{\prime} \dot{\alpha}^{\prime}} \bar{\omega}_{\mu}{ }^{a 2}, \\
\epsilon^{\mu \nu} \mathcal{D}_{0 \mu} \psi_{0 \nu+}^{\alpha \dot{\alpha}} & =-2 m \epsilon^{\mu \nu} \gamma_{0 \mu} \psi_{0 \nu-}^{\alpha \dot{\alpha}}, \\
\epsilon^{\mu \nu} \mathcal{D}_{0 \mu} \psi_{0 \nu-}^{\alpha^{\prime} \dot{\alpha}^{\prime}} & =2 m \epsilon^{\mu \nu} \gamma_{0 \mu} \psi_{0 \nu+}^{\alpha^{\prime} \dot{\alpha}^{\prime}} .
\end{aligned}
$$

Eq. (5.39) also shows that the third term in eq. (5.36) can be written as

$$
\begin{gathered}
\frac{1}{2 \epsilon} i \epsilon^{\mu \nu}\left(\bar{\epsilon}_{0+\alpha \dot{\alpha}} \gamma_{0 \nu} \psi_{0 a+}^{\alpha \dot{\alpha}}-\bar{\epsilon}_{0-\alpha^{\prime} \dot{\alpha}^{\prime}} \gamma_{0 \nu} \psi_{0 a-}^{\alpha^{\prime} \dot{\alpha}^{\prime}}-\bar{\epsilon}_{0+\alpha \dot{\alpha}} \gamma_{a} \psi_{0 \nu+}^{\alpha \dot{\alpha}}+\bar{\epsilon}_{0-\alpha^{\prime} \dot{\alpha}^{\prime}} \gamma_{a} \psi_{0 \nu}^{\alpha^{\prime} \dot{\alpha}^{\prime}}\right) \bar{\omega}_{\mu}^{a 2} \\
+4 i m^{2} \epsilon^{\mu \nu}\left(\bar{\epsilon}_{0-\alpha \dot{\alpha}} \gamma_{0 \mu} \psi_{0 \nu-}^{\alpha \dot{\alpha}}-\bar{\epsilon}_{0+\alpha^{\prime} \dot{\alpha}^{\prime}} \gamma_{0 \mu} \psi_{0 \nu+}^{\alpha^{\prime} \dot{\alpha}^{\prime}}\right)
\end{gathered}
$$

The terms proportional to $\bar{\omega}_{\mu}^{a 2}$ in this equation, in turn, can be written as

$$
\frac{1}{2 \epsilon} i \epsilon^{\mu \nu}\left(\bar{\epsilon}_{0+\alpha \dot{\alpha}} \gamma_{0 \nu} \psi_{0 \mu+}^{\alpha \dot{\alpha}}-\bar{\epsilon}_{0-\alpha^{\prime} \dot{\alpha}^{\prime}} \gamma_{0 \nu} \psi_{0 \mu^{-}}^{\alpha^{\prime} \dot{\alpha}^{\prime}}\right) \bar{\omega}_{a}^{a 2}=4 m^{2} \delta_{Q} e_{0} g_{0}^{\mu \nu} g_{(2) \mu \nu}
$$

which cancels the last term in eq. (5.37). On the other hand, by using eq. (5.39) the remaining terms in eq. (5.40) become

$$
-2 i m \epsilon^{\mu \nu}\left[\bar{\epsilon}_{0-\alpha \dot{\alpha}} \mathcal{D}_{0 \mu} \psi_{0 \nu+}^{\alpha \dot{\alpha}}+\bar{\epsilon}_{0+\alpha^{\prime} \dot{\alpha}^{\prime}} \mathcal{D}_{0 \mu} \psi_{0 \nu-}^{\alpha^{\prime} \dot{\alpha}^{\prime}}\right] .
$$


Collecting these results and after some more algebra we finally obtain the supertransformation of the total action as

$$
\delta_{Q} S=\frac{1}{4 m} i \int d^{2} x \tilde{e} \overline{\tilde{\eta}}_{\alpha \dot{\alpha}} \tilde{\gamma}^{\mu \nu} \tilde{\psi}_{\mu \nu}^{\alpha \dot{\alpha}}
$$

where $\tilde{\psi}_{\mu}^{\alpha \dot{\alpha}}$ is the field strength of the Rarita-Schwinger fields defined in eq. (3.21).

To summarize, our results of anomalies for the local symmetry transformations are given by eqs. (5.31), (5.32), (5.35) and (5.43). There is no anomaly in twodimensional general coordinate, local Lorentz, vector $\mathrm{SU}(2)$ gauge and local super transformations. The remaining symmetries, i.e., two-dimensional Weyl, axialvector gauge and super Weyl symmetries, are anomalous. The anomalies (5.31), (5.32), (5.35) and (5.43) form an anomaly supermultiplet [26], in the sence that they transform to one another by the two-dimensional local supertransformation. This can be shown by using the Wess-Zumino consistency condition [27, 28] and the absence of the two-dimensional local supersymmetry anomaly. These anomalies correspond to the $(4,4)$ super Virasoro algebras with the central charge $c=\frac{6 \pi}{m}=\frac{3 l}{2 G}$. Anomalies in the $(p, q)$ theories for other values of $p, q=1,2,4$ can be obtained from these results by truncation of the fields discussed at the end of sect. 2 .

We would like to thank M. Natsuume for informing us of ref. 113. This work is supported in part by the Grant-in-Aid for Scientific Research on Priority Area 707 "Supersymmetry and Unified Theory of Elementary Particles", Japan Ministry of Education.

\section{References}

[1] J. Maldacena, The large $N$ limit of superconformal field theories and supergravity, Adv. Theor. Math. Phys. 2 (1998) 231, hep-th/9711200.

[2] S.S. Gubser, I.R. Klebanov and A.M. Polyakov, Gauge theory correlators from noncritical string theory, Phys. Lett. B428 (1998) 105, hep-th/9802109.

[3] E. Witten, Anti de Sitter space and holography, Adv. Theor. Math. Phys. 2 (1998) 253, hep-th/9802150. 
[4] S. Ferrara, C. Frønsdal and A. Zaffaroni, On $N=8$ supergravity on $\mathrm{AdS}_{5}$ and $N=4$ superconformal Yang-Mills theory, Nucl. Phys. B532 (1998) 153, hep-th/9802203.

[5] H. Liu and A.A. Tseytlin, $D=4$ super Yang-Mills, $D=5$ gauged supergravity and $D=4$ conformal supergravity, Nucl. Phys. B533 (1998) 88, hepth/9804083.

[6] M. Nishimura and Y. Tanii, Supersymmetry in the AdS/CFT correspondence, Phys. Lett. B446 (1999) 37, hep-th/9810148.

[7] A. Achúcarro and P.K. Townsend, A Chern-Simons action for threedimensional anti-de Sitter supergravity theories, Phys. Lett. B180 (1986) 89.

[8] M. Henningson and K. Skenderis, The holographic Weyl anomaly, JHEP 07 (1998) 023, hep-th/9806087.

[9] S. Hyun, W.T. Kim and J. Lee, Statistical entropy and AdS/CFT correspondence in BTZ black holes, Phys. Rev. D59 (1999) 084020, hep-th/9811005.

[10] S. Nojiri and S.D. Odintsov, Conformal anomaly for dilaton coupled theories from AdS/CFT correspondence, Phys. Lett. B444 (1998) 92, hep-th/9810008; On the conformal anomaly from higher derivative gravity in AdS/CFT correspondence, hep-th/9903033.

[11] C.R. Graham and E. Witten, Conformal anomaly of submanifold observables in AdS/CFT correspondence, hep-th/9901021.

[12] O. Aharony, J. Pawełczyk, S. Theisen and S. Yankielowicz, A note on anomalies in the AdS/CFT correspondence, hep-th/9901134.

[13] V. Balasubramanian and P. Kraus, A stress tensor for anti-de Sitter gravity, hep-th/9902121.

[14] M. Pernici and P. van Nieuwenhuizen, A covariant action for the SU(2) spinning string as a hyperkähler or quaternionic nonlinear sigma model, Phys. Lett. B169 (1986) 381.

[15] A. Strominger and C. Vafa, Microscopic origin of the Bekenstein-Hawking entropy, Phys. Lett. B379 (1996) 99, hep-th/9601029. 
[16] C.G. Callan and J.M. Maldacena, D-brane approach to black hole quantum mechanics, Nucl. Phys. B472 (1996) 591, hep-th/9602043.

[17] J. Maldacena and A. Strominger, $\mathrm{AdS}_{3}$ black holes and a stringy exclusion principle, JHEP 12 (1998) 005, hep-th/9804085.

[18] J. de Boer, Six-dimensional supergravity on $\mathrm{S}^{3} \times \mathrm{AdS}_{3}$ and $2 \mathrm{~d}$ conformal field theory, hep-th/9806104.

[19] J.D. Brown and M. Henneaux, Central charges in the canonical realization of asymptotic symmetries: an example from three dimensional gravity, Commun. Math. Phys. 104 (1986) 207.

[20] M. Henningson and K. Sfetsos, Spinors and the AdS/CFT correspondence, Phys. Lett. B431 (1998) 63, hep-th/9803251.

[21] G.E. Arutyunov and S.A. Frolov, On the origin of supergravity boundary terms in the AdS/CFT correspondence, hep-th/9806216.

[22] M. Henneaux, Boundary terms in the AdS/CFT correspondence for spinor fields, hep-th/9902137.

[23] G.W. Gibbons and S.W. Hawking, Action integrals and partition functions in quantum gravity, Phys. Rev. D15 (1977) 2752.

[24] L. Susskind and E. Witten, The holographic bound in anti-de Sitter space, hep-th/9805114.

[25] B. Zumino, Y.-S. Wu and A. Zee, Chiral anomalies, higher dimensions, and differential geometry, Nucl. Phys. B239 (1984) 477.

[26] M.T. Grisaru, Anomalies in supersymmetric theories, in Recent Developments in Gravitation, eds. M. Levy and S. Deser (Plenum Press, 1979).

[27] J. Wess and B. Zumino, Consequences of anomalous Ward identities, Phys. Lett. B37 (1971) 95.

[28] Y. Tanii, The structures of anomalies in supersymmetric string theories, Nucl. Phys. B289 (1987) 187. 